\title{
Photophysics of size-selected InP nanocrystals: Exciton recombination kinetics
}

\author{
S.-H. Kim, R. H. Wolters, and J. R. Heath \\ Molecular Design Institute, Lawrence Berkeley Laboratory and Department of Chemistry and Biochemistry, \\ University of California, Los Angeles, Los Angeles, California 90095-1569
}

(Received 17 June 1996; accepted 2 August 1996)

\begin{abstract}
We report here on the size-dependent kinetics of exciton recombination in a III-V quantum dot system, InP. The measurements reported include various frequency dependent quantum yields as a function of temperature, frequency dependent luminescence decay curves, and time-gated emission spectra. This data is fit to a three-state quantum model which has been previously utilized to explain photophysical phenomena in II-VI quantum dots. The initial photoexcitation is assumed to place an electron in a (delocalized) bulk conduction band state. Activation barriers for trapping and detrapping of the electron to surface states, as well as activation barriers for surface-state radiationless relaxation processes are measured as a function of particle size. The energy barrier to detrapping is found to be the major factor limiting room temperature band-edge luminescence. This barrier increases with decreasing particle size. For $30 \AA$ particles, this barrier is found to be greater than $6 \mathrm{~kJ} / \mathrm{mol}$ - a barrier which is more than an order of magnitude larger than that previously found for $32 \AA$ AdS nanocrystals. (C) 1996 American Institute of Physics. [S0021-9606(96)00942-7]
\end{abstract}

\section{INTRODUCTION}

In this paper, we utilize photoluminescence (PL) to elucidate the kinetics of the various photophysical processes corresponding to excited-state relaxation within a sizeselected, surface passivated III-V nanocrystal system, InP. The object of this work is not only to measure the relevant kinetic parameters of the exciton recombination pathways, but also to evaluate these results within the context of the well-studied II-VI quantum dot systems. Thus, a brief account of the relevant aspects of II-VI quantum dot photophysics is presented in this Introduction.

Nanocrystals of II-VI semiconducting materials have provided a prototype for the investigation of quantum size effects. ${ }^{1,2}$ These particles may be prepared as high quality single crystals characterized by very nearly discrete size distributions, and with diameters ranging from below $20 \AA$ to near $120 \AA^{3} .^{3}$ The first excited electronic state of these particles occurs at an energy higher than the bulk band gap, and, for the smaller particles, the electronic states are observed to be discrete, rather than continuous. Of particular interest has been the spectroscopy and dynamics of the optically prepared excited states. Much of the motivation for these investigations has arisen from the possibility of exploiting quantum dots for photonics-based applications. In principle, it should be possible to create a series of photoemitters, each chemically identical (varying only by size), which luminesce throughout the visible and near-IR regions of the spectrum. Critical to such applications are questions related to the efficiency of band-edge luminescence. For example, what is the role that surface states play in the dynamics of exciton recombination, and can various surface passivation schemes be employed to modify that role? A three-state model has been successfully utilized by a number of research groups to explain various aspects of the PL spectra of certain II-VI nanocrystals. ${ }^{4-6}$ In the model, the three "states" correspond to the valence and conduction band (VB and $\mathrm{CB}$ ) manifolds, and localized surface states. Although the details of II-VI semiconductor nanocrystals are quite complex and not completely worked out, ${ }^{7,8}$ certain basic facts have emerged. Photoexcitation initially places an electron in an (interior) $\mathrm{CB}$ state. At temperatures near $0 \mathrm{~K}, \mathrm{CB}$-edge luminescence provides the primary mechanism for exciton recombination. Trapping of the excited electron to a surface state is a slightly activated process, and at some finite temperature, such trapping begins to dominate the photophysics. Above a few degrees Kelvin, the surface states provide a reservoir for almost all subsequent band-edge or surface state emission processes. Band-edge emission, then, proceeds through an activated "detrapping" mechanism, which returns an electron from a surface state back to the conduction band edge. The activation barriers for detrapping are typically much greater than for trapping, and this is reflected in the temperature dependence of the PL spectrum.

By comparison with the II-VI's, the III-V class of nanocrystals have received only a small amount of attention, in spite of the relative importance of III-V's in semiconductor photonics applications. This is largely due to the lack of synthetic schemes for producing III-V nanocrystals that are of sufficient quality to facilitate reliable photophysics investigations. However, within the last couple of years, syntheses have appeared in the literature which describe the preparation of various III-V nanocrystals systems, including GaAs, ${ }^{9}$ InP ${ }^{10} \operatorname{In}_{x} \mathrm{Ga}_{1-x} \mathrm{P}$, and $\mathrm{GaP} .{ }^{11}$ We have recently reported on the preparation and fundamental physical properties of sizeselected, surface passivated, monodisperse InP nanocrystals. $^{12}$ Our synthesis, which is a modification of a scheme reported by the Nozik group, produces nanocrystals that, although not near the state-of-the-art defined for IIVI's, are of suitable quality for investigating InP nanocrystals size-dependent photophysics. 
In this paper, we investigate the temperature and frequency dependence, and the temporal evolution of the photoluminescence from 49,43 , and $30 \AA$ diameter surfacepassivated InP nanocrystals. We find that the three-state model which has been applied to II-VI quantum dots provides an excellent framework for understanding the excited state dynamics in InP nanocrystals. However, we also find that the kinetic parameters which describe the various relaxation processes are quite different from those reported for various II-VI systems. For example, the "detrapping" activation energy is found to increase from $<4.0$ to $>6 \mathrm{~kJ} / \mathrm{mol}$ with decreasing particle size. These numbers are more than a factor of 10 times larger than were previously reported for similarly sized CdS nanocrystals. ${ }^{4}$

\section{EXPERIMENT}

\section{A. Particle synthesis}

The InP nanocrystals used in these experiments were synthesized using the dehalosylation reaction developed by the Wells group. ${ }^{13}$ The general reaction scheme was described in our previous paper, ${ }^{12}$ so only a brief description of the synthesis is presented here. All syntheses were carried out using standard glove-box, vacuum line, and other airless techniques. A measured amount of $\mathrm{InCl}_{3}$ is heated to $100{ }^{\circ} \mathrm{C}$ in trioctylphosphineoxide (TOPO) for $12 \mathrm{~h}$ to make an $(\mathrm{InCl})_{x}(\mathrm{TOPO})_{y}$ complex. An equimolar amount of $\mathrm{P}\left[\mathrm{Si}\left(\mathrm{CH}_{3}\right)_{3}\right]_{3}$ is added to the stirring reaction mixture, and the mixture is heated slowly to a temperature of $265^{\circ} \mathrm{C}$, where it is allowed to react for a period of several days. At this point, nanocrystalline InP, capped with TOPO, is the primary product. For the experiments discussed here, the TOPO cap was partially replaced with dodecylamine (DDA). To do this, the reaction mixture is cooled to $100{ }^{\circ} \mathrm{C}$, and an excess of DDA is added to the reaction flask. The mixture is kept stirring at $100{ }^{\circ} \mathrm{C}$ for an additional 3 days. The reaction mixture is then cooled to room temperature, and the particles are sizeselectively precipitated from the reaction mixture by the addition of a fixed amounts of methanol. Using this technique, the largest particles precipitate first, and up to 40 optically unique particle size distributions can be prepared from a single synthesis. The individual distributions are characterized by distribution widths near $20 \%$.

Following the size-selective precipitation, the particles are dissolved in methylcyclohexane (MCH) (99\% spectrophotometric grade, Aldrich). $\mathrm{MCH}$ forms an organic glass at low temperatures, and thus makes an excellent matrix for cryogenic experiments. In our previous work, we correlated room temperature PL measurements with X-ray photoelectron spectroscopy (XPS) to demonstrate the effect of oxidation on PL efficiency. ${ }^{12}$ We determined that at least partial surface oxidation was necessary for particle luminescence. The particles are fairly stable in air, only exhibiting measurable amounts of oxidation after a day or two of air exposure. Thus, to ensure that the particles would luminesce with reasonable efficiency, the particles were stored in air for a couple of days prior to use. However, to ensure a consistency between the various samples, all measurements were carried out within five days of particle synthesis.

\section{B. Photoluminescence measurements}

For the temperature-dependent PL measurements, InP nanocrystals (in $\mathrm{MCH}$ ) were transferred into a liquid-cell mounted onto the cold finger of a Helix CTI Cryogenics closed-cycle He refrigerator. The liquid cell consists of two sapphire wafers separated by an aluminum spacer and mounted between copper plates. A temperature sensing diode was attached to the aluminum spacer. The cell temperature was varied from 15 to $300 \mathrm{~K}$ through the use of a Scientific Instruments temperature controller.

Continuous wave $(\mathrm{CW})$ PL spectra were taken using the (chopped) $568 \mathrm{~nm}$ line of a Coherent Innova 70 multigas $\left(\mathrm{Ar}^{+} / \mathrm{Kr}^{+}\right)$ion laser for excitation. The collected emission was passed through a $1 \mathrm{~nm}$ bandwidth notch filter designed to remove the excitation frequency, dispersed in a monochromator (Oriel Multispec 257) and detected with a Hamamatsu R928 photomultiplier tube. The signal was processed through a Stanford SR510 lock-in amplifier. All spectra were corrected using a calibrated source. Intensities of emission spectra were normalized by comparison with a Rhodamine $6 \mathrm{G}$ dye standard.

For time-resolved emission experiments, we used a frequency doubled $10 \mathrm{~Hz} Q$-switched Nd:YAG pulsed laser (Quanta Ray DCR-2A) as an excitation source. Laser pulses $(<50 \mu \mathrm{J}, 8 \mathrm{~ns}$ FWHM) were sent to the sample through a $400 \mu \mathrm{m}$ multimode optical fiber. Emitted photons were collected and detected with the same monochromator and PMT. The signal was gated and processed using Stanford Instruments (SR 250) Gated Integrator and Boxcar Averager. Time gated spectra were recorded by setting an appropriate gate width (discussed later), and scanning the monochromator from 550 to $800 \mathrm{~nm}$ in 2-4 nm increments and collecting 200-300 laser shots per increment. Emission decay traces were recorded by Tektronix TDS 520A oscilloscope. A small portion of the excitation laser beam was directed onto a photodiode, to provide a trigger for the scope or the boxcar integrator.

The absorption spectra of the InP nanocrystals were taken at room temperature using a Perkin-Elmer Lambda 3B UV/vis spectrophotometer. In our previous work, we correlated particle size (as determined by $\mathrm{x}$-ray powder diffraction line broadening and transmission electron microscopy) with the lowest energy feature in the room temperature absorption spectrum. ${ }^{12}$ This correlation was utilized to extract the sizes of the particles investigated here from their UV/vis spectra.

\section{RESULTS AND DISCUSSION}

\section{A. The kinetic model}

The object of this work is to elucidate the size-dependent rates and activation energies for various photophysical processes in InP nanocrystals, and thus enable a direct comparison of this III-V nanocrystal system with the well-studied II-VI quantum dots. Figure 1 shows the room temperature 


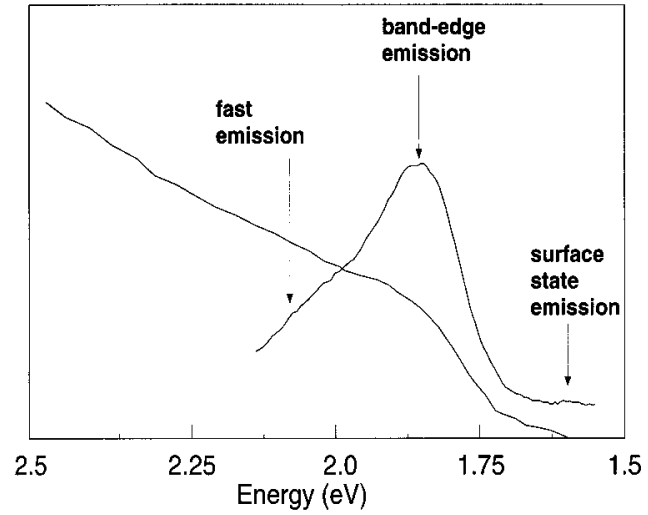

FIG. 1. Room temperature absorption and luminescence spectra of $43 \AA$ InP particles.

absorption and emission spectra of $43 \AA$ InP nanocrystals. Labeled on the figure are three emission components which correspond to the separate recombination processes that are active at room temperature. The three-state model which describes these processes is presented in Fig. 2. This is the model which, as discussed above, has been used in previous descriptions of II-VI nanocrystal photophysics. Rate constants and arrows corresponding to various relaxation pathways are labeled on the figure and defined below. The straight arrows correspond to mechanisms resulting in photon emission, and the curved arrows indicate dark processes.

Upon absorption of a photon, the excited state has two possibilities for relaxation. $k_{f}$ describes an extremely fast (subnanosecond) emission pathway in which the excited state immediately relaxes back to the initial state. This process does not exhibit a temperature dependence. Bawendi and co-workers used polarization studies to assign a lifetime of $\sim 65$ ps to this process in $32 \AA$ CdSe nanocrystals, ${ }^{6}$ and similar numbers $(80 \mathrm{ps})$ have more recently been reported by Chamarro and co-workers. ${ }^{14} k_{t}$ and $k_{d}$ are the rate constants for the activated processes of surface trapping and detrapping, respectively. The barrier for surface trapping is ex-

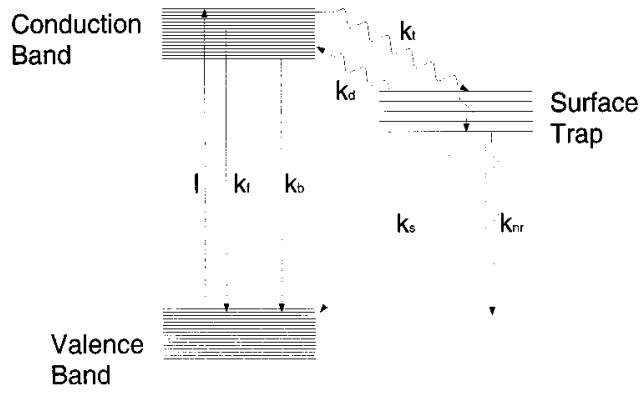

FIG. 2. The three-level model for excited state relaxation in semiconductor quantum dots. Initial photoexcitation places an electron in a bulk (delocalized) conduction band state, and the process is labeled $I$. The relaxation processes are: $k_{f}$-fast emission rate; $k_{b}$-band edge emission rate; $k_{t}$-trapping rate; $k_{d}$-detrapping rate; $k_{s}$-surface trap emission rate; and $k_{\mathrm{nr}}$-nonradiative decay rate. Processes resulting in the absorption or emission of a photon are represented by straight lines, and nonradiative processes are labeled with curved lines. pected to be small, and this process should dominate the room temperature photophysics such that the surface states will act as reservoirs for all subsequent relaxation processes.

An electron confined to a surface state has three possibilities for relaxation to the ground state. $k_{d}$ corresponds to the above-described activated detrapping process, and is expected to exhibit a strong temperature dependence. $k_{s}$ is the rate constant for exciton recombination via surface state photoemission. Surface state emission should exhibit a complex temperature dependence, since it is a function of $k_{d}, k_{s}$, and $k_{\mathrm{nr}}$ (all activated processes). $k_{\mathrm{nr}}$ is the rate constant for the (activated and nonactivated) radiationless relaxation processes from the surface states. These and possibly other dark processes provide the dominant relaxation pathways throughout the temperature regime explored here $(\sim 15-300$ $\mathrm{K})$, as the total quantum yield never rises above $1 \%$.

A mathematical model describing the photophysical processes outlined in Fig. 2 may be constructed. Assuming that every process follows first order kinetics, and employing a steady state approximation, one can find

$$
\begin{aligned}
& Q Y_{b} \propto\left[A^{*}\right] k_{b}=\frac{\left(k_{d}+k_{\mathrm{nr}}+k_{s}\right) k_{b} I}{k_{t} k_{\mathrm{nr}}+k_{t} k_{s}+k_{b} k_{d}+k_{b} k_{\mathrm{nr}}+k_{b} k_{s}}, \\
& Q Y_{s} \propto\left[A^{s}\right] k_{s}=\frac{k_{t} k_{s} I}{k_{t} k_{\mathrm{nr}}+k_{t} k_{s}+k_{b} k_{d}+k_{b} k_{\mathrm{nr}}+k_{b} k_{s}}, \\
& Q Y_{b} / Q Y_{s} \propto \frac{k_{b}\left(k_{d}+k_{\mathrm{nr}}+k_{s}\right)}{k_{t} k_{s}},
\end{aligned}
$$

where $Q Y_{b}$ and $Q Y_{s}$ represent quantum yields for band edge and surface trap emission, respectively, and all rate constants are defined in Fig. 2. $\left[A^{*}\right]$ and $\left[A^{S}\right]$ refer to the excited state population within the $\mathrm{CB}$ and surface-state manifold, respectively.

We assume that only $k_{t}, k_{d}$, and $k_{\mathrm{nr}}$ are functions of $T$ and will follow Arrhenius behavior. It is also reasonable to assume that the activation barrier for trapping $\left(\epsilon_{t}\right)$ is very small, and therefore, will not exhibit much of a temperature dependence in the $15-300 \mathrm{~K}$ temperature range studied here. If the rate constants for $k_{d}$ and $k_{\mathrm{nr}}$ are then given by: $k_{d}=A_{d} \exp \left(-\epsilon_{d} / T\right)$ and $k_{\mathrm{nr}}=A_{\mathrm{nr}} \exp \left(-\epsilon_{\mathrm{nr}} / T\right)$, then Eq. (3) becomes

$$
Q Y_{b} / Q Y_{s}=C_{1} \exp \left(-\epsilon_{d} / T\right)+C_{2} \exp \left(-\epsilon_{\mathrm{nr}} / T\right)+C_{3} .
$$

Figure 3 shows PL spectra of three different sizes of InP samples at room temperature and near $20 \mathrm{~K}$. Three general features are observed in the luminescence spectra, and are labeled $\lambda_{f}, \lambda_{b}$, and $\lambda_{s}$. Prior to fitting these spectra to the above described kinetic model, we will discuss these features qualitatively. The highest energy feature is relatively insensitive to temperature, does not exhibit a size dependence, and is characterized by the shortest lifetime (faster than our ability to measure) of the three components. It is therefore assigned to the $k_{f}$ process. Band-edge emission, denoted with a pair of dashed lines, and labeled $\lambda_{b}$, is observed to lie just below the absorption edge for each spectrum (see Fig. 1). This emission corresponds to the $k_{b}$ process. At low tem- 
(a)
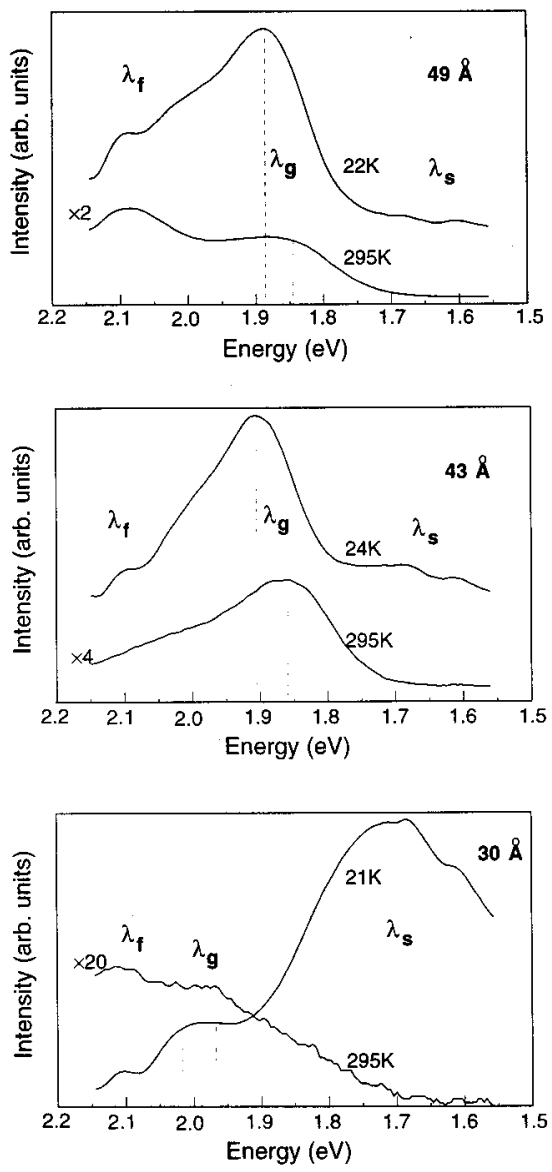

FIG. 3. Continuous wave photoluminescence spectra for three different size InP particles at room temperature and $\sim 20 \mathrm{~K}$. (a) $49 \AA$, (b) $43 \AA$, (c) $30 \AA$. The excitation frequency is $568 \mathrm{~nm}(2.18 \mathrm{eV})$. The various $\lambda_{i}$ labels correspond to the fast, band-edge, and surface photoemission components as described in Fig. 2 and in the text.

peratures it shifts to higher energies by approximately 0.045 $\mathrm{eV}$ and sharpens, presumably due decreasing phonon interactions. This emission also exhibits a particle size dependence, as expected. The emission signal recorded at lower photon energies $\left(\lambda_{s}\right)$ is assigned to surface trap photoemission (the $k_{s}$ process). The relative intensity of surface trap emission increases for smaller particles, presumably due to the larger surface to core ratio. Similar effects for surface trap emission has been reported for the case of $\mathrm{CdS} .^{5}$

\section{B. Temperature dependent PL: Activation barriers}

In Fig. 4, total quantum yields are plotted as a function of temperature for the $43 \AA$ particles. Similar curves were collected for the other two particle sizes. The luminescence intensity is not a simple function of temperature. Instead, several different processes are involved in emission kinetics. In order to elucidate the parameters relevant to the kinetic model discussed above, it is necessary to separately measure the quantum yields of both the surface-trap luminescence and the band-edge luminescence. According to Eq. (4), it is

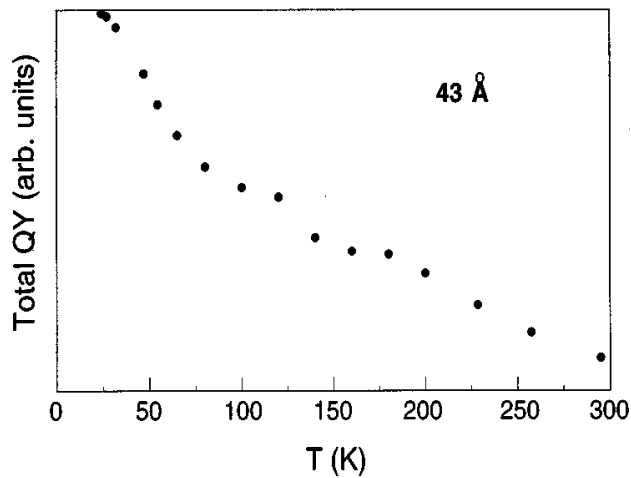

FIG. 4. Total photoluminescence quantum yields for $43 \AA$ InP particles as a function of temperature. Note that the plot does not follow a simple functional form, and that the total quantum yield is beginning to saturate at very low temperature. This was seen for all particle sizes, and is related to the nonradiative relaxation processes beginning to shut down as temperature is lowered. According to the model, at temperatures of a few K, the surfacestate trapping process should also be closed and the quantum yields should exhibit a sharp increase.

the ratio of these two yields which contains information concerning the barriers for the surface trapping and the radiationless processes.

Figure 5 shows the measured quantum yields for three particle sizes, plotted as the ratio $Q Y_{b} / Q Y_{s}$. In the higher temperature regime, these data are fitted reasonably well by a single exponential, and such a fit is included in the figure. This implies a much smaller pre-exponential factor for the nonradiative process than for the detrapping process, and thus $\epsilon_{d}$ dominates the observed temperature dependence. For the $43 \AA$ particles, a second exponential corresponding to the $k_{\mathrm{nr}}$ process was also fit to the ratio data. Indeed, the fitted pre-exponential factor corresponding to this process $\left(C_{2}\right)$ was a factor of 50 less than that for the $k_{d}$ process $\left(C_{1}\right)$. The fitted activation energies for the detrapping process are 3.6(0.5), 5.4(1.2), and 6.3(1.0) kJ/mol for 49,43 , and $30 \AA$ particles, respectively. There are a few things to note about these values. This activation barrier should somewhat reflect the energy difference between the surface states and the edge of the CB. The observation that $\epsilon_{d}$ increases with decreasing particle size implies that the InP nanocrystal surface states are more localized than the CB-edge states, and therefore, are less sensitive to changing particle size. Thus, the smaller particles are characterized by increasingly deeper surface traps.

The $\epsilon_{d}$ values reported here are more than an order of magnitude higher than the $0.27 \mathrm{~kJ} / \mathrm{mol}$ barrier reported by the McLendon group for $32 \AA \mathrm{CdS}$ nanocrystals. ${ }^{4}$ Increased ionic bonding character within a bulk crystal leads to successively shallower surface traps. ${ }^{15}$ For the relatively ionic II-VI class of materials, the case of surface states on bulk $\mathrm{ZnO}$ has been well studied. According to Luth, ${ }^{16}$ the presence of the surface (termination of the bulk periodic potential), and the effects of surface reconstruction are only weak perturbations when compared to the strong ionic forces which dominate the bonding, and thus the surface states remain very shallow. This argument obviously does not hold 
(a)

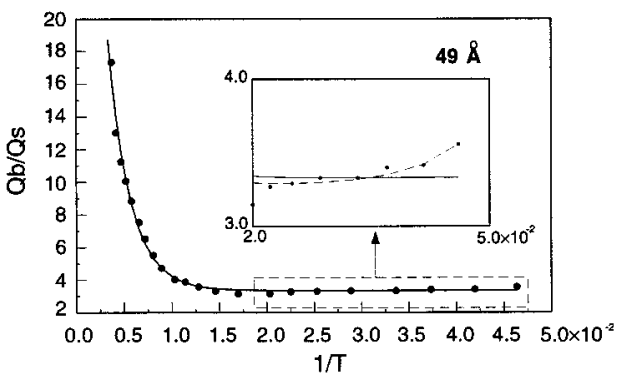

(b)

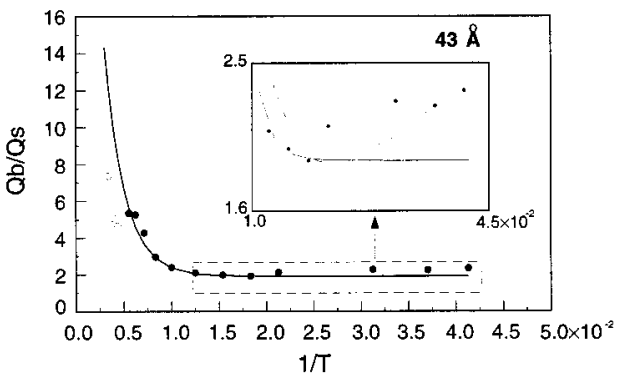

(c)

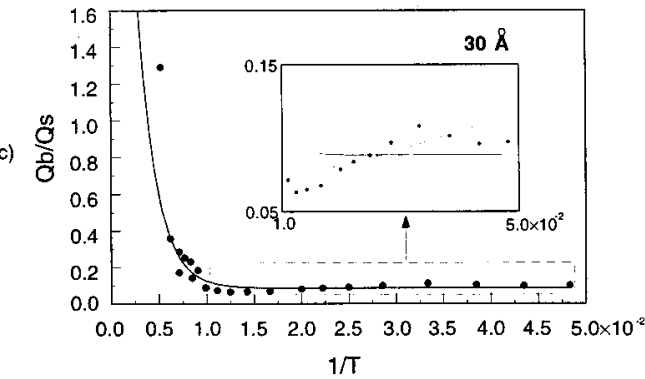

FIG. 5. Quantum yields ratio between band edge emission and surface trap emission. (a) $49 \AA$, (b) $43 \AA$, (c) $30 \AA$. This data was fit successfully to just the first term of Eq. (4), and the fit is shown as a solid line. The activation barrier for detrapping $\left(\epsilon_{d}\right)$ was extracted from this fit. In the boxes, the data at low temperatures is magnified to show a slight increase in the quantum yield ratio. Solid lines in those boxes are the same fits described above, and dashed lines are fits to Eq. (5), which considers the trapping process to be activated. The empty circles in the plot for the $43 \AA$ particles have very large $(>50 \mathrm{~K})$ statistical uncertainties in temperature. For this reason, the activation barriers extracted from the surface trap emission quantum yields are a little more reliable.

for covalently bound materials. Therefore, given the comparatively more covalent character of the bonding in III-V's, it is not surprising that the surface states are deeper than are observed for II-VI nanocrystals. These deeper traps, and thus the increased $\epsilon_{d}$, may be the major factor which limits the PL quantum yield of InP nanocrystals. Emission quantum yields for II-VI semiconductors vary from synthesis to synthesis, but are generally reported to be around 5\% or more. The maximum quantum yield for our InP particles, on the other hand, is only about $0.5 \%$ at $20 \mathrm{~K}$.

Close inspection of the data in Fig. 5 indicates that the ratio of the quantum yields for band gap and surface state PL actually exhibits a slight increase at lower temperatures. This implies that the trapping process $\left(k_{t}\right)$ is weakly activated and the temperature dependence of $k_{t}$ may not be completely neglected in the low temperature regime studied here. Thus, including the trapping process and neglecting the radiation- less processes in Eq. (4), the new description of the quantum yield ratios becomes

$$
Q Y_{b} / Q Y_{s}=C_{1} \exp \left(-\epsilon_{d} / T\right)+C_{4} \exp \left(\epsilon_{t} / T\right)+C_{5} .
$$

Fits to the data which take an activated $k_{t}$ process into account are included in the insets of Fig. 5. Trapping activation barriers ranging from 100 to $200 \mathrm{~J} / \mathrm{mol}$ were extracted from these fits. These numbers have large error bars, so we cannot determine whether $\epsilon_{t}$ exhibits a size dependence. These barriers correspond to temperatures in the range of $20 \mathrm{~K}$ or less, and imply that, at very low temperatures, the surface trapping channel should be closed, and the PL quantum yield should greatly increase.

As discussed above, the single exponential fits shown in Fig. 5 imply that the pre-exponential factor $\left[C_{2}\right.$ of Eq. (4) $]$ describing the radiationless process is much smaller than $C_{1}$. This can be rationalized through first order perturbation theory. The matrix element connecting a surface state with an interior state most likely increases with decreasing particle size. Nevertheless, a phonon can provide a perturbation with significantly strengthens this coupling. However, within the context of perturbation theory, the coupling is going to decrease as the energy difference between the two states increases. This is the major difference between the preexponential factors $C_{1}$ and $C_{2}$. $C_{1}$ is related to the electronphonon coupling of two states very close in energy, while $C_{2}$ is related to the electron-phonon coupling of two states quite far apart in energy. Thus the single exponential fits in Fig. 5 are not surprising. Nevertheless, the radiationless processes do exhibit a temperature dependence which is discernible in the PL spectra. Figure 6 presents plots of the surface state quantum yields as a function of temperature for all three particle sizes. These measurements correspond to Eq. (2). Two separate fits to the data are included in the Figure, and each fit includes certain assumptions about Eq. (2). For both fits the weakly activated $k_{t}$ process is assumed to be a constant of temperature, while $k_{d}$ is considered to be an activated process. For the dashed-line fit, $k_{\mathrm{nr}}$ is assumed to be constant with temperature. In the solid-line fit, the $k_{\mathrm{nr}}$ process is assumed to be activated, and the activation energy $\left(\epsilon_{\mathrm{nr}}\right)$ for that process fitted. $\epsilon_{\mathrm{nr}}$ is observed to decrease with decreasing particle size, from about $1.0 \mathrm{~kJ} / \mathrm{mol}$ for $49 \AA$ particles, to about $0.6 \mathrm{~kJ} / \mathrm{mol}$ for $30 \AA$ particles. However, the uncertainties in the $\epsilon_{\mathrm{nr}}$ measurements are large, so the trend is not statistically significant. Nevertheless, it is interesting to note that the temperature dependent nonradiative processes are closed at low temperature. This is the reason for the saturation behavior observed in the total quantum yields (Fig. 4) at low temperature.

\section{Time resolved photoluminescence: Rate constants}

In order to get the real time information on the kinetic processes, we measured time resolved PL spectra for $37 \AA$ particles at $15 \mathrm{~K}$. In Figure 7, we compare the emission spectra for different time windows with the $\mathrm{CW}$ spectrum. The emission observed within and shortly after the time window of the excitation pulse is near $2.1 \mathrm{eV}$. This emission 
(a)

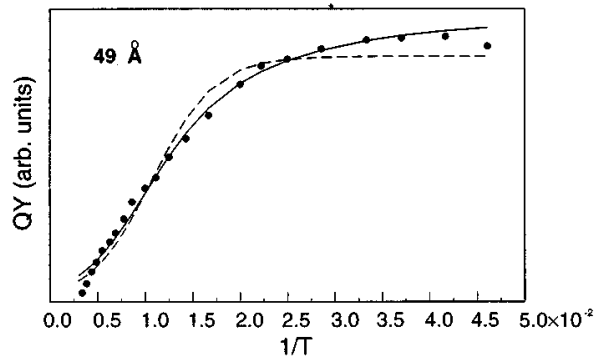

(b)
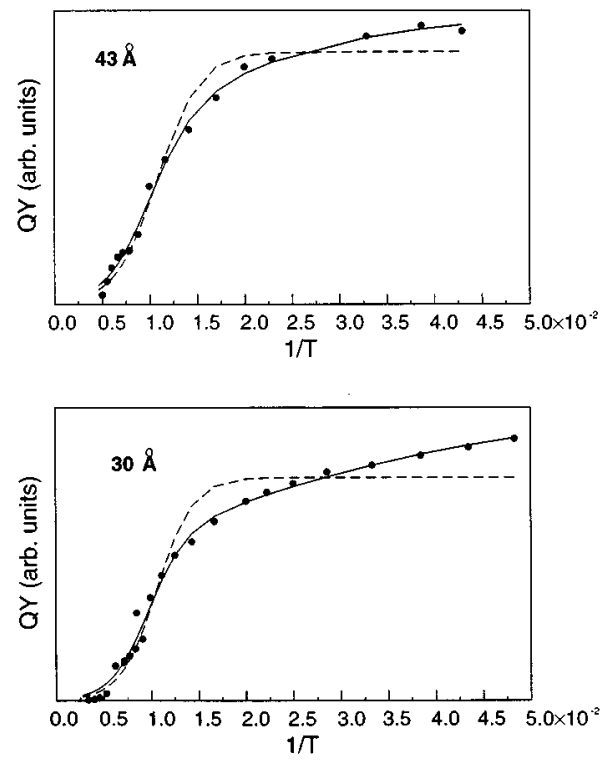

FIG. 6. Quantum yields for surface trap emission as a function of temperature the three particle sizes. Solid lines represent a fit to Eq. (2) that includes the temperature dependence of nonradiative relaxation process. Dashed lines are fits to Eq. (2) which consider that process to be temperature independent.

actually extends to $2.3 \mathrm{eV}$, although the higher energy component is removed by a $550 \mathrm{~nm}(2.26 \mathrm{eV})$ long pass filter designed to eliminate the excitation wavelength. We assign this feature to be the fast $\left(k_{f}\right)$ component described above. In the second spectrum, the fast component has decreased in intensity, and a second feature near the band gap energy is growing. This luminescence is assigned to the $k_{b}$ process. The third spectrum, recorded after a delay of several microseconds, reveals the slow component that we have assigned to the $\mathbf{k}_{s}$ process.

Figure 8 shows emission decay curves at three wavelengths. The decay at $555 \mathrm{~nm}(2.24 \mathrm{eV})$, corresponding to the $k_{f}$ component, is faster $(<8 \mathrm{~ns})$ than the temporal resolution of our spectrometer. At $615 \mathrm{~nm}(2.02 \mathrm{eV})$ and $750 \mathrm{~nm}(1.65$ $\mathrm{eV})$ representing $k_{b}$ and $k_{s}$ process have tails lasting $\sim 2 \mu \mathrm{s}$ and $>10 \mu$ s, respectively.

Rate constants estimated from this data, and the various fitted activation energies, are summarized in Table I.

\section{CONCLUSIONS}

A three state electronic structure model which has been previously used to describe certain aspects of exciton recombination kinetics in II-VI nanocrystal systems is extended here to a III-V nanocrystal system, InP. Various rate laws (a)

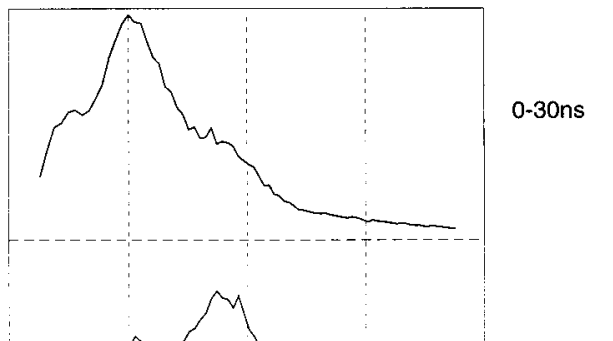

(c)

(b)

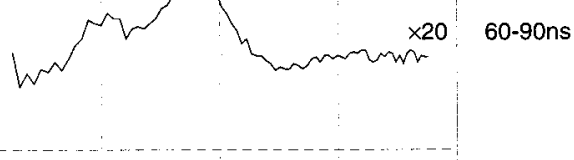

(d)

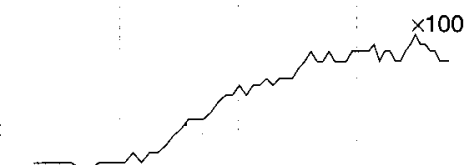

5 usec with $60 \mathrm{~ns}$ averaging time window

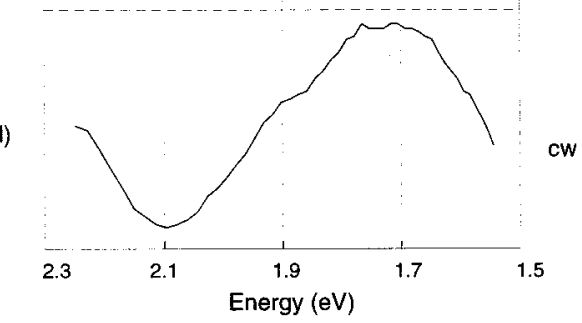

FIG. 7. Time resolved PL spectra for $37 \AA$ InP particles at $15 \mathrm{~K}$. Absorption edge at room temperature is at $1.89 \mathrm{eV}$. For the continuous wave spectrum, $514.5 \mathrm{~nm}(2.41 \mathrm{eV})$ line of $\mathrm{Ar}^{+}$laser is used as an excitation source. The gate width and step size for spectra (a) and (b) is $30 \mathrm{~ns}$ and $2 \mathrm{~nm}$, respectively. For spectrum (c), these numbers are $60 \mathrm{~ns}$ and $4 \mathrm{~nm}$.

are extracted from the kinetic model and are used to fit photoluminescence data. Activation energies for $\mathrm{CB}$ to surfacestate trapping, surface-state to $\mathrm{CB}$ detrapping, and surfacestate nonradiative relaxation are extracted from temperature and wavelength dependent photoluminescence quantum yields. In general, the model is found to provide an excellent framework for understanding exciton recombination processes in InP nanocrystals. A major result from this work is that the surface states in InP nanocrystals are deeper and less

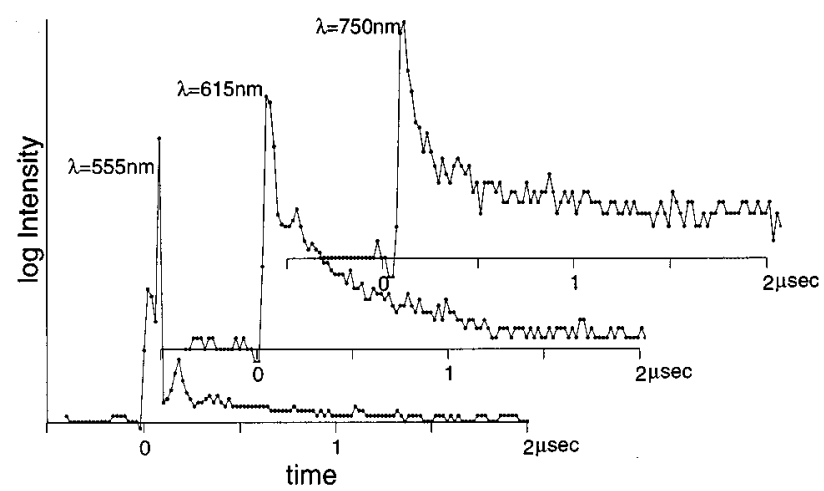

FIG. 8. Emission decay at three different wavelengths, 555, 615, and 750 $\mathrm{nm}$. The long tail in $750 \mathrm{~nm}$ curve extends in time to beyond $10 \mu \mathrm{s}$. 
TABLE I. Activation barriers and rate constants for the three level kinetic model. All numbers in $\mathrm{kJ} / \mathrm{mol}$; Tabulated uncertainties represent $90 \%$ confidence limits. n.f.—not fitted. n.m. - not measured.

\begin{tabular}{ccccccc}
\hline \hline Particle size & $\epsilon_{d}$ & $\epsilon_{\mathrm{nr}}$ & $\epsilon_{t}$ & $k_{f}(20 \mathrm{~K})$ & $k_{b}(20 \mathrm{~K})$ & $k_{s}(20 \mathrm{~K})$ \\
\hline $49 \AA$ & $3.6(0.5)^{\mathrm{a}}$ & $1.0(7)^{\mathrm{a}}$ & n.f. $^{\mathrm{a}}$ & $<8 \mathrm{~ns}$ & n.m. & n.m. \\
& $4.6(2)^{\mathrm{b}}$ & n.f. $^{\mathrm{b}}$ & $0.10(6)^{\mathrm{b}}$ & & & \\
$43 \AA$ & $5.4(1.2)^{\mathrm{a}}$ & $0.94(4.0)^{\mathrm{a}}$ & n.f. $^{\mathrm{a}}$ & $<8 \mathrm{~ns}$ & $\sim 2 \mu \mathrm{s}$ & $\sim 10 \mu \mathrm{s}$ \\
& $5.4(6)^{\mathrm{b}}$ & $0.96(3.0)^{\mathrm{b}}$ & $0.18(5)^{\mathrm{b}}$ & & & \\
$30 \AA$ & $6.3(1.0)^{\mathrm{a}}$ & $0.6(5.0)^{\mathrm{a}}$ & n.f. $^{\mathrm{a}}$ & $<8 \mathrm{~ns}$ & n.m. & n.m. \\
& $11.1(2.0)^{\mathrm{b}}$ & n.f. $^{\mathrm{b}}$ & $0.22(8)^{\mathrm{b}}$ & & & \\
\hline \hline
\end{tabular}

${ }^{a}$ Derived from fit to temperature dependent surface PL quantum yields.

${ }^{\mathrm{b}}$ Derived from fit to temperature dependent ratio of band-edge/surface PL quantum yields.

sensitive to size than are observed for the case of the more ionic II-VI class of nanocrystals. As a consequence, the activation barriers corresponding to the surface-state $\rightarrow$ conduction band detrapping process are nearly an order of magnitude larger than have been observed for II-VI quantum dots.

In our previous work, we were able to correlate total photoemission quantum yields with the oxidation of surface $\mathrm{P}$ atoms. At the time of this writing, we do not yet have a clear indication of the detailed chemical nature of these surface states, or what role, if any, that indium atoms play in surface trapping. We are currently addressing these question by correlating ${ }^{31} \mathrm{P}$ NMR spectra together with surface oxidation and photoluminescence yields.

High activation barriers for detrapping lead directly to low band-edge photoluminescence quantum yields $(<0.5 \%)$ at all but the very lowest of temperatures. Although surface passivation with various other organic or organometallic surfactants than were used here may increase PL efficiency in InP nanocrystals, any such effect is likely to be small. However, solution-phase epitaxial growth of a high band-gap material, such as $\mathrm{GaP}$, onto the surface of the InP nanocrystals may provide a route toward dramatically increasing PL efficiency. We are currently investigating the feasibility of such chemistry.

\section{ACKNOWLEDGMENTS}

This work was supported by the Office of Naval Research, Order No. N00014-95-F-0099 and by the Director, Office of Energy Research, Office of Basic Energy Research, Division of Materials Sciences, of the U.S. Dept. of Energy
Under Contract No. DE-AC03-76SF00098. J.R.H. acknowledges support from the David and Lucile Packard Foundation.

${ }^{1}$ N. Chestnoy, T. D. Harris, R. Hull, and L. E. Brus, J. Phys. Chem. 90, 3393 (1986).

${ }^{2}$ Y. Wang and N. Herron, J. Phys. Chem. 91, 257 (1987).

${ }^{3}$ C. B. Murray, D. J. Norris, and M. G. Bawendi, J. Am. Chem. Soc. 115, 8706 (1993).

${ }^{4}$ M. O'Neil, J. Marohn, and G. McLendon, J. Phys. Chem. 94, 4356 (1990).

${ }^{5}$ A. Eychmuller, A. Hasslebarth, L. Katsikas, and H. Weller, J. Lumin. 48, 745 (1991).

${ }^{6}$ (a) M. G. Bawendi, W. L. Wilson, L. Rothbert, P. J. Carroll, T. M. Jedju, M. L. Steigerwald, and L. E. Brus, Phys. Rev. Lett. 65, 1623 (1990); (b) M. G. Bawendi, P. J. Carroll, W. L. Wilson, and L. E. Brus, J. Chem. Phys. 96, 946 (1992).

${ }^{7}$ J. J. Shiang, A. V. Kadavanich, R. K. Grubbs, and A. P. Alivisatos, J. Phys. Chem. 99, 595 (1995).

${ }^{8}$ D. J. Norris and M. G. Bawendi, J. Chem. Phys. 103, 5260 (1995).

9 (a) M. A. Olshavsky, A. N. Goldstein, and A. P. Alivisatos, J. Am. Chem. Soc. 112, 9438 (1990); (b) H. Uchida, C. J. Curtis, P. V. Kamat, K. M. Jones, and A. J. Nozik, J. Phys. Chem. 96, 1156 (1992).

${ }^{10}$ (a) O. I. Micic, C. J. Curtis, K. M. Jones, J. R. Spragure, and A. J. Nozik, J. Phys. Chem. 98, 4966 (1994); (b) M. D. Dvorak, B. L. Justus, D. K. Gaskill, and D. G. Henershot, Appl. Phys. Lett. 66, 804 (1995).

${ }^{11}$ O. I. Micic, J. R. Sprague, C. J. Curtis, K. M. Jones, J. L. Machol, and A. J. Nozik, J. Phys. Chem. 99, 7754 (1995).

${ }^{12}$ A. Guzellian, J. Bowen-Katari, A. Kadavanich, U. Benin, A. P. Alivisatos, R. Wolters, C. C. Arnold, and J. R. Heath, J. Phys. Chem. 100, 7212 (1996).

${ }^{13}$ R. L. Wells, C. G. Pitt, A. T. McPhail, A. P. Purdy, S. Shafieezad, and R. B. Hallock. Chem. Mater. 1, 4 (1989).

${ }^{14}$ M. Chamarro, C. Gourdon, P. Lavallard, O. Lublinskaya, and A. I. Ekimov, Phys. Rev. B 55, 1336 (1996).

${ }^{15} \mathrm{M}$. Lannoo, in Handbook of Surfaces and Interfaces, edited by L. Dobrzynski (Garland STPM, New York, 1978), pp. 11-98.

${ }^{16}$ H. Luth, Surfaces and Interfaces of Solids (Springer, New York, 1993). 